\title{
A Western single-center experience with endoscopic submucosal dissection for early gastrointestinal cancers
}

\author{
Sergio Coda ${ }^{1,2}$, Paolo Trentino ${ }^{1}$, Fabio Antonellis $^{1}$, Barbara Porowska $^{1}$, Francesco Gossetti $^{3}$, \\ Franco Ruberto ${ }^{4}$, Francesco Pugliese ${ }^{4}$, Giulia d’Amati ${ }^{5}$, Paolo Negro $^{3}$, and Takuji Gotoda ${ }^{6}$ \\ ${ }^{1}$ Endoscopy Unit, Department of Surgery "Paride Stefanini," University of Rome "La Sapienza," Rome, Italy \\ ${ }^{2}$ Department of Medicine, Division of Experimental Medicine, Imperial College London, Room 731, Blackett Laboratory, South Kensington \\ Campus, Prince Consort Road, London SW7 2AZ, UK \\ ${ }^{3}$ General Surgery Unit, Department of Surgery "Paride Stefanini," University of Rome "La Sapienza," Rome, Italy \\ ${ }^{4}$ Critical Care Medicine and Pain Therapy, Department of Anesthesiology, University of Rome "La Sapienza," Rome, Italy \\ ${ }^{5}$ Department of Experimental Pathology, University of Rome "La Sapienza," Rome, Italy \\ ${ }^{6}$ Department of Endoscopy, National Cancer Center Hospital, Tokyo, Japan
}

\begin{abstract}
Endoscopic submucosal dissection (ESD) has gained worldwide acceptance as a treatment for early gastrointestinal cancers (EGICs). However, the management of these tumors in the Western world is still mainly surgical. Our aim was to evaluate the safety and feasibility of ESD at a European center. Based on the knowledge transferred by one of the most experienced Japanese institutions, we conducted a pilot study on 25 consecutive patients with EGICs located in the esophagus $(n=3)$, stomach $(n=7)$, duodenum $(n=1)$, and colon $(n=14)$ at our tertiary center over a 2-year-period. The main outcome measurements were complete (R0) resection, as well as en-bloc resection and the management of complications. The $\mathrm{RO}$ and en-bloc resection rates were $100 \%$ and $84 \%$, respectively. There were three cases of bleeding and five cases of perforation. With a median follow up of 18 months, two recurrences were observed. We conclude that ESD for early esophageal and gastric cancers is feasible and effective, while colonic ESD requires more expertise.
\end{abstract}

Key words Gastrointestinal neoplasms · Endoscopic submucosal dissection · Endoscopic gastrointestinal surgery

\section{Introduction}

In the era of minimally invasive therapy, endoscopic submucosal dissection (ESD) has gained wide acceptance in Asian countries for the treatment of early gastrointestinal cancers (EGICs) [1-3].

In the West, ESD is rarely reported [4-8], both because of the apparently lower incidence of EGICs and because of the much rarer usage of this technique.

Offprint requests to: $\mathrm{S}$. Coda

Received: October 13, 2009 / Accepted: January 28, 2010
We report a single-center exploratory program involving 25 cases of ESD for the treatment of EGICs, performed at "Paride Stefanini" Surgical Department, University of Rome "La Sapienza," Rome, Italy.

\section{Patients and Methods}

\section{Patients}

Twenty-five unselected consecutive patients (10 men and 15 women; mean age, 70 years; range, $47-88$ years) with EGICs underwent ESD at our institution from April 2007 to September 2009. There were 3 esophageal, 7 gastric, 1 duodenal, and 14 colonic lesions (Table 1 ). The mean size of the lesions was $25 \mathrm{~mm}$ (range, $10-50 \mathrm{~mm}$ ). Inclusion criteria followed those of Oyama [9] for lesions of the esophagus; the extended Gotoda criteria for the stomach [10]; and laterally spreading tumors (LSTs) greater than $20 \mathrm{~mm}$ in diameter [11] or Paris 0-II type lesions for the colon.

\section{Preoperative evaluation}

Diagnostic endoscopy included contrast chromoscopy with $0.2 \%$ indigo carmine dye or $2.5 \%$ Lugol solutions, or narrow band imaging (NBI) inspection, together with magnifying endoscopy (110 to 150x; Olympus GIFQ160Z or CFQ160Z; Olympus, Tokyo, Japan). Histological assessment confirmed the lesions as low-grade or high-grade noninvasive neoplasia (LGNIN or HGNIN).

All the patients were informed by oral and written explanation about the planned treatment. They were aware of the level of experience in ESD of the two operators (S.C. and P.T.). This study was conducted in accordance with the ethical principles of the Declaration of Helsinki and without any external financial support. 


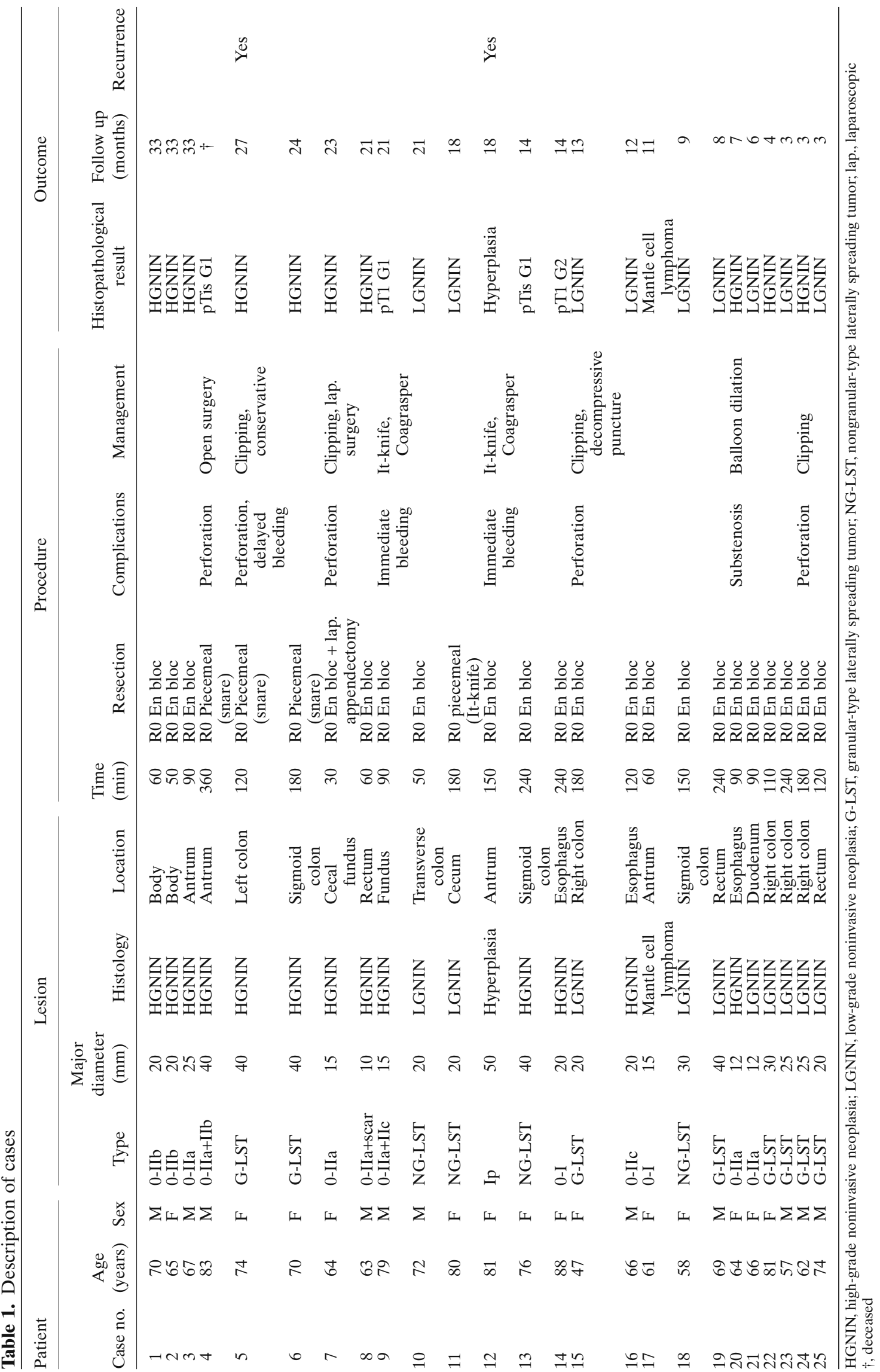




\section{ESD technique}

An It-knife, Hook-knife, or Dual-knife (Olympus Medical Systems, Tokyo, Japan) was used for dissection (Olympus PSD-60; Endocut mode, effect 3, 60- to 80-Watt cutting, 20-Watt forced coagulation), after multiple injections of saline and epinephrine solution (1: 20 000), and staining with indigo-carmine, and further repeated injections of hyaluronic acid or $10 \%$ glycerol. An attachment cap was regularly used in order to facilitate the dissection.

In cases of failed en-bloc removal, final excision was achieved using a polypectomy snare in a piecemeal fashion.

At the end, careful inspection of the ulcer site and coagulation of borders and visible vessels was routinely performed.

\section{Outcomes and management}

The main outcomes of the study were the feasibility of ESD, data on the percentages of en-bloc and complete resections, and an indication of various possible complications.

Feasibility was indicated by the number of ESDs successfully accomplished.

All the patients were operated on in the operating theater, under propofol sedation, with a surgical team available to treat possible complications not manageable endoscopically. The day after the ESD, patients had plain abdominal X-rays to rule out perforation, and in the absence of bleeding signs, they were allowed a liquid diet 24-48 h after the procedure. Proton pump inhibitors were routinely administered for 1 month in patients with gastric lesions only.

\section{Follow-up}

Follow up (FU) with biopsies included 1-, 3- and 12-month examinations. Recurrence was defined as the presence of pathological tissue at the resection site.

\section{Complications}

Minor bleeding was immediately treated with repeated injections of saline solution or with Coagrasper forceps (Olympus Medical Systems).

Perforation was defined as the endoscopic intraoperative diagnosis of a hole in the viscus, or the presence of free air on plain X-ray the day after the procedure, with or without clinical signs. All identified perforations were treated intraoperatively by the apposition of clips (Resolution Clip; Boston Scientific, Natick, MA, USA).

\section{Histopathological assessment}

Specimens were fixed and orientated on a board. After fixation, the samples were sectioned at $2-\mathrm{mm}$ intervals parallel to the closest resection margin to assess both lateral and vertical margins. Multiple histological sections (at least 10) were obtained from each paraffin block, stained with hematoxylin \& eosin (Figs. 1 and 2).

\section{Results}

En-bloc resection was feasible in $3 / 3$ esophageal, 6/7 gastric, 1/1 duodenal, and 10/14 colonic cases (overall $84 \%$ ). Complete resection was achieved in all the en-bloc procedures. Median operating time was $120 \mathrm{~min}$ (range, 30-360 min). The median follow up was 18 months (range, 3-33 months).

Compared to the preoperative diagnosis, histological examination of the whole sample resulted in six upstagings and one downstaging.

Overall, bleeding occurred in $12 \%$ of the patients and perforation in $20 \%$. Esophageal substenosis occurred in one patient. Disease recurrence was present in 2 patients.

\section{Discussion}

Japanese experts recommend that surgeons perform ESD on gastric lesions first, as they are technically easier to remove and exhibit a lower rate of complications compared to esophageal or colonic lesions $[10$, 12-14].

Nevertheless, in the present study, it was decided to include all consecutive unselected cases referred to our Department. This was a research program aimed at assessing the feasibility of ESD as an alternative treatment for EGICs at a University Hospital with an estimated catchment area of about 1000000 inhabitants.

S.C. attended the National Cancer Center Hospital $(\mathrm{NCCH})$ in Tokyo for 3 months, to gain practice before carrying out the first autonomous procedure. For the first month of his training, he observed at least three to five complete procedures per day performed by T.G. and his team. He then started to practise on isolated pig stomachs, performing about three ESDs per week. In the third month, he performed, under the strict supervision of T.G., 3 ESDs in three patients with EGCs. Throughout this period, he followed the team in all their clinical duties, including the management of complications.

P.T. has 26 years of experience in therapeutic and emergency endoscopy. He observed at least 30 videos of ESDs performed by T.G. and his colleagues.

The first 3 procedures in the present study were performed by S.C. supervised by T.G. Then, S.C. performed 14 procedures autonomously and assisted P.T. in performing 2 procedures. The last 6 ESDs were performed by P.T. autonomously. 

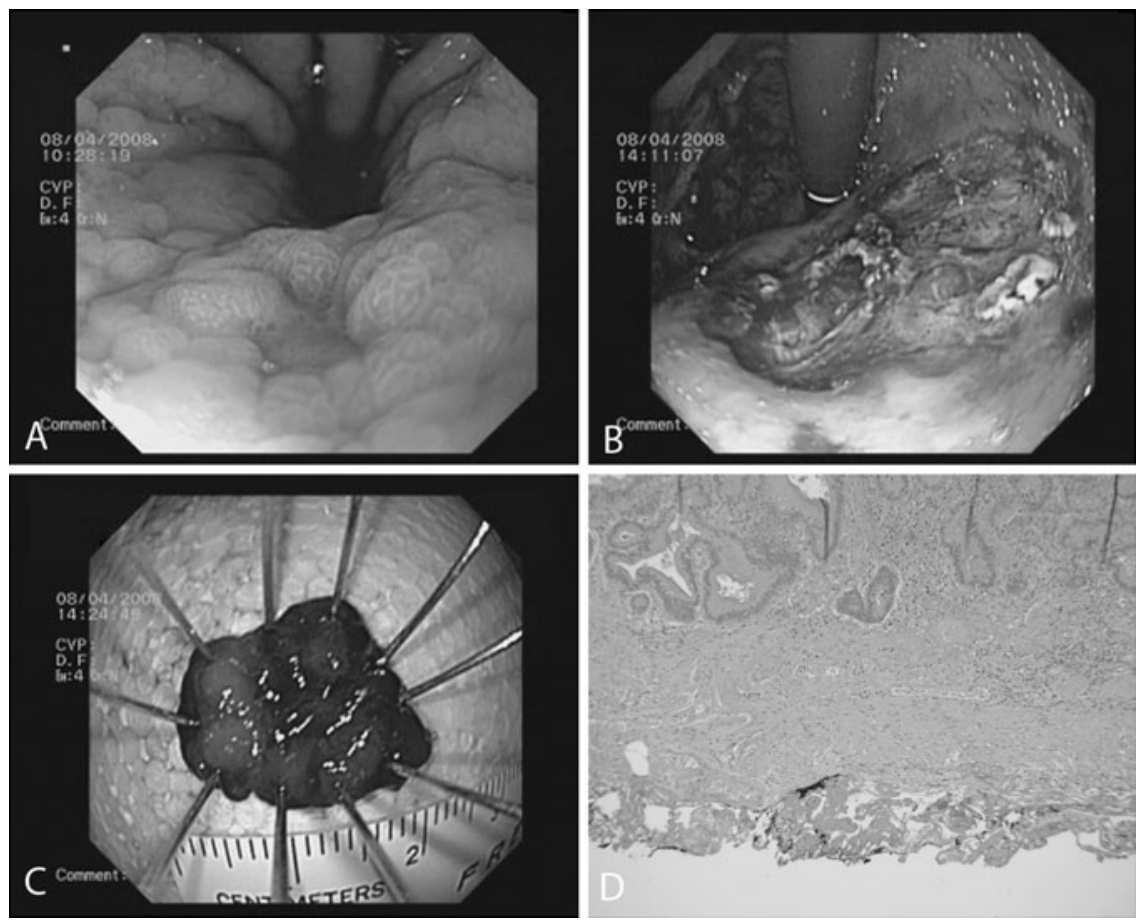

Fig. 1. A Early gastric cancer (0-IIa + IIc type; $15 \mathrm{~mm}$ ), of the fundus, along the lesser curvature just below the cardia, after staining with indigo-carmine. B Residual ulcer after endoscopic submucosal dissection. C En-bloc excised lesion fixed and orientated on a board for histopathological assessment. D Well-differentiated (G1) intramucosal adenocarcinoma of intestinal type, vertical margins free of tumor. $\mathrm{H} \& \mathrm{E}, \times 20$
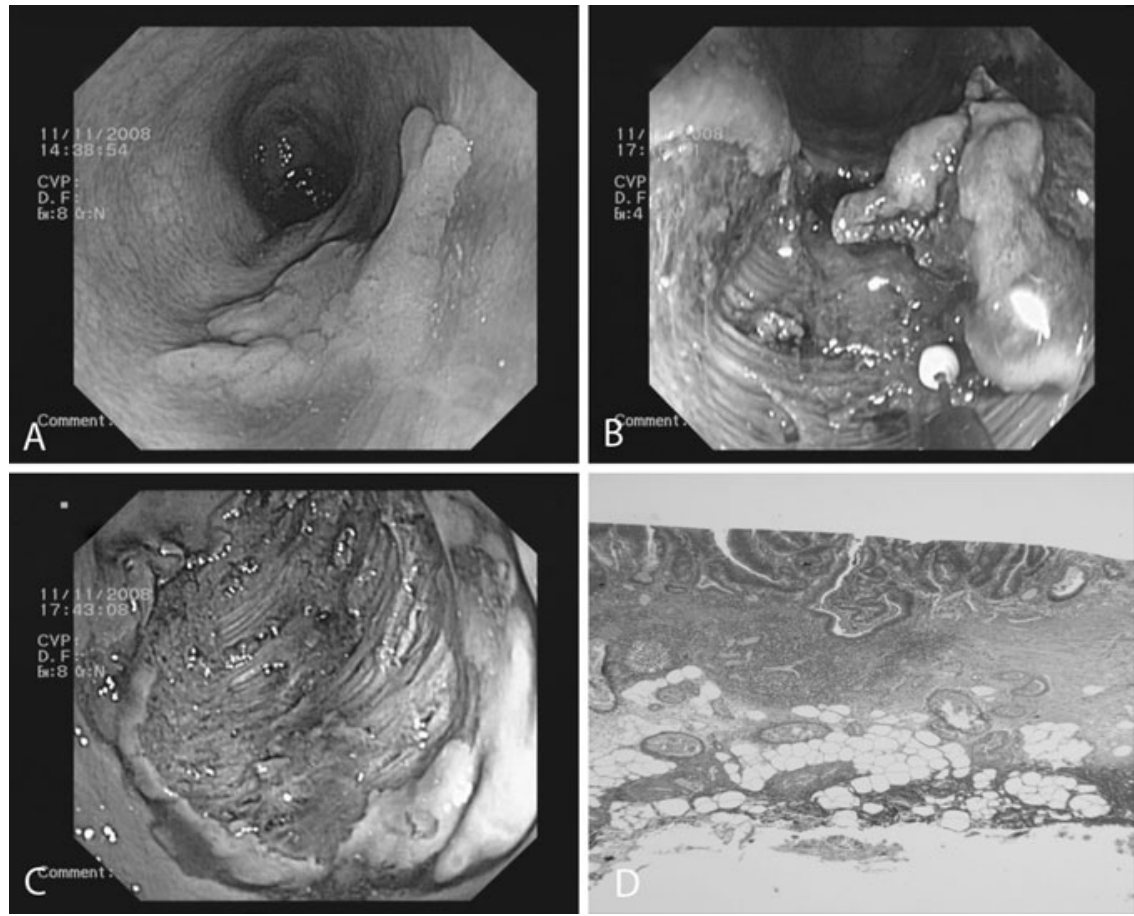

Fig. 2. A Laterally spreading tumor (nongranular type, $40 \mathrm{~mm}$ ) of the sigmoid colon. B Dissection of the submucosal layer with an It-knife. C. En-bloc excised lesion fixed and orientated on a board for histopathological assessment. D. Welldifferentiated (G1) adenocarcinoma. The lesion is largely intramucosal, with focal infiltration of the muscularis mucosae. $\mathrm{H}$ \& E, $\times 10$

EGC is the most accepted indication for ESD worldwide. In Western countries, only a few series have been published recently, with encouraging results [15-17].

Overall, we had a satisfactory en-bloc and complete resection rate for upper gastrointestinal (GI) cancers $(90 \%)$, while the rate was lower for colonic series $(71 \%)$.
In the literature, the perforation rate ranges between $1 \%$ and $10 \%$ [11, 18-21]. There was a high perforation rate in our small colonic series, probably because of the initial experience with ESD and the unselected cases. However, all of the perforations were recognized at the time of operation, and subsequently clipped. Only one 

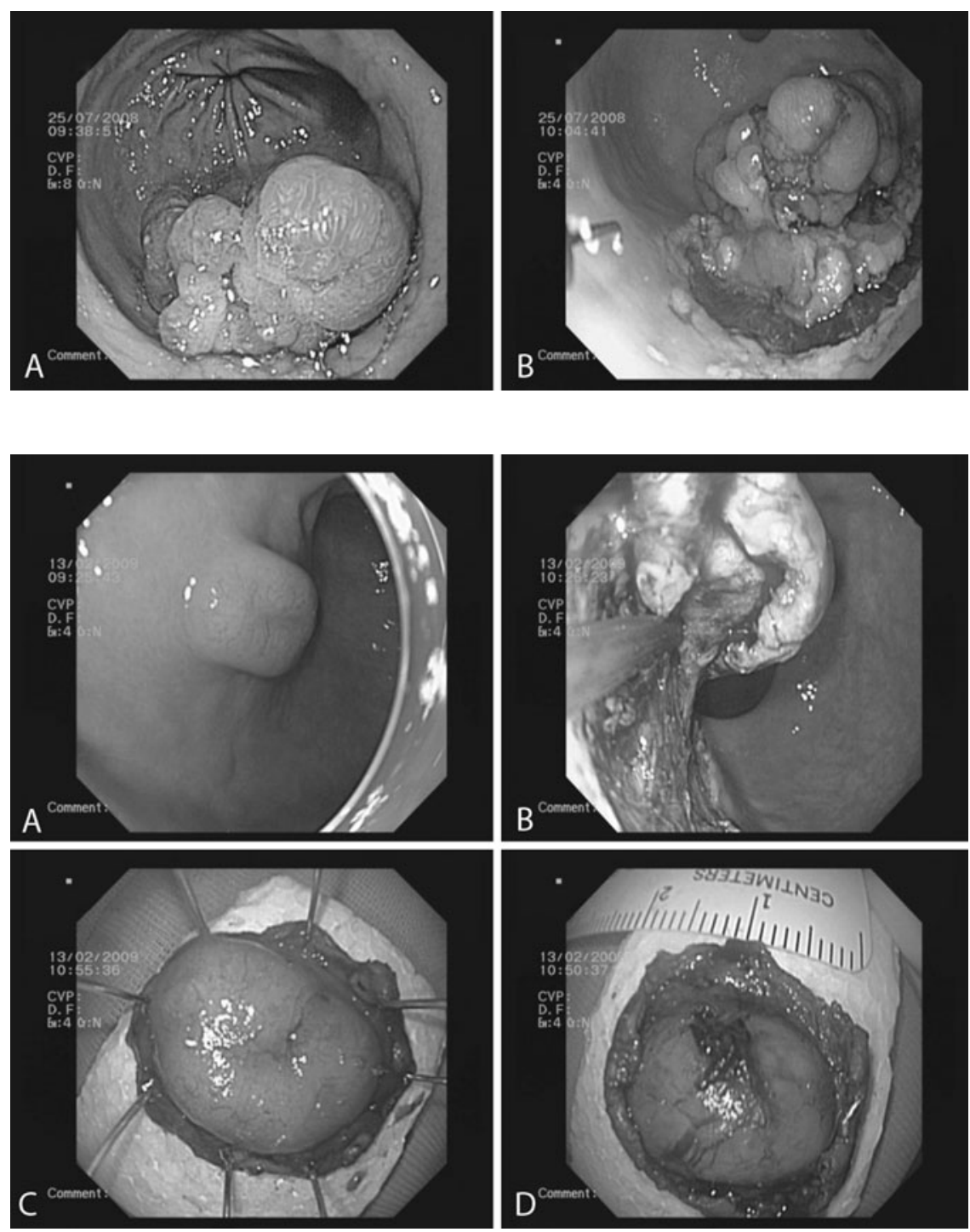

Fig. 3. A Giant recurrent hyperplastic polyp of the gastric antrum (50 mm), after staining with indigo-carmine. B Circumferential incision performed with a Hook-knife around the polyp
Fig. 4. A Solitary gastric nodule of recurrent localized mantle cell lymphoma. B Submucosal dissection performed with an It-knife. C En-bloc excised lesion fixed and orientated on a board for histopathological assessment (mucosal side). D En-bloc excised lesion fixed and orientated on a board for histopathological assessment (submucosal side) patient received an abdominal ultrasound (US)-guided needle decompression.

Open surgery was required in one patient only (a gastric case), an 83-year-old patient with a large EGC $(40 \mathrm{~mm})$, at the beginning of our study. Twenty-four hours after the procedure, a small amount of free air was seen on the plain X-ray, but an emergency laparotomy failed to disclose evidence of perforation. The patient died 40 days later of respiratory insufficiency, presumably as a consequence of the laparotomy.

Substenosis occurred after one esophageal ESD, in a patient with HGNIN within an 8-cm-long segment of circumferential Barrett's esophagus; the substenosis was effectively managed by endoscopic balloon dilation.

Recurrence was present in one giant $(50 \mathrm{~mm})$ recurrent antral hyperplastic polyp, responsible for severe anemia. Suspecting neoplastic progression, we performed an en-bloc and complete ESD (Fig. 3). At the 3-month FU endoscopy, again a recurrence (10-mm) was noted. EUS was then performed, but without any clear explanation for this recurrence. This patient was retreated with a standard polypectomy and argon plasma coagulation.

One gastric case was a solitary antral nodule, which was a recurrent localized mantle cell lymphoma This patient, who had received chemotherapy (CHT) and bone marrow transplantation, was offered an ESD as alternative treatment to further CHT (Fig. 4). She was disease-free at the 6-month endoscopic control.

\section{Conclusions}

Our study has many limitations: a limited and heterogeneous cohort, with and without prior endoscopic treatment, with and without neoplasia; two operators with variable experience and learning curves; and 
limited FU for several patients. However, we have demonstrated the successful cooperation and transfer of practical knowledge between two tertiary Institutions with hitherto differing therapeutic approaches.

Following this preliminary experience, ESD has been introduced in our hospital clinical practice as an experimental alternative treatment to surgery for early GI cancers.

In summary, we believe that:

- At least 30 procedures should be carefully observed for each location.

- At least ten ESDs should be performed on animal models before starting with patients (stomach only), and at least 3 initial procedures should be supervised by foreign experts.

- Colonic ESD should be performed in the lower rectum first; the right colon should be attempted only after adequate experience has been gained.

- Initial experiences should be gained at specialized centers. Caution is mandatory, and tight cooperation with a surgical team is advisable.

ESD for early esophageal and gastric cancers is feasible and effective.

Colonic ESD requires considerably more training than ESD for early esophageal and gastric cancers, due to the high risk of perforation; also, more cooperation with our expert Asian colleagues is needed to achieve the expertise appropriate for facing difficult lesions.

A more extended study is necessary to confirm the results of the small scale study presented here, and more studies in the West are needed to identify full guidelines for the use of this technique.

Acknowledgments S.C. thanks all those who participated in his training. Special thanks go to Dr. Jonathan P.J. White for his valuable guidance and assistance in writing this article.

\section{References}

1. Chiu PWY. Endoscopic submucosal dissection-bigger piece, better outcome! Gastrointest Endosc 2006;64:884-5.

2. Chung IK, Lee JH, Lee SH, Kim SJ, Cho JH, Cho WY, et al. Therapeutic outcomes in 1000 cases of endoscopic submucosal dissection for early gastric neoplasms: Korean ESD Study Group multicenter study. Gastrointest Endosc 2009;69:122835.

3. Yahagi N. Is esophageal endoscopic submucosal dissection an extreme treatment modality, or can it be a standard treatment modality? Gastrointest Endosc 2008;68:1073-5.
4. Coda S, Lee S-Y, Gotoda T. Endoscopic mucosal resection and endoscopic submucosal dissection as treatments for early gastrointestinal cancers in Western Countries. Gut and Liver 2007; $1: 12-21$.

5. Coda S, Oda I, Gotoda T, Saito Y. EMR and ESD for early gastrointestinal cancers. In: Conio M, Siersema P, Repici A, Ponchon T, editors. Endoscopic mucosal resection. Oxford, UK: Blackwell; 2008. p. 185-96.

6. Nehaus H, Costamagna G, Deviere J, Fockens P, Ponchon T, Rosch T. Endoscopic submucosal dissection (ESD) of early gastric lesion using a new double-channel endoscope (the "R-scope"). Endoscopy 2006;38:1016-23.

7. Probst A, Arnholdt H, Anthuber M, Messmann H. Endoscopic submucosal dissection (ESD) of large mucosal and submucosal lesions in the GI tract. Experience in the western world (abstract). Gastrointest Endosc 2007;65:A 278.

8. Deprez P.H, Aouattah T, Yeung C-PR, Fiasse RH, Horsmans Y, Piessevaux H. Endoscopic resection of superficial gastric tumors (abstract). Gastrointes Endosc 2007;65:A 164.

9. Oyama T. Endoscopic submucosal dissection for esophageal cancer. In: Niwa H, supervising editor. New challenges in gastrointestinal endoscopy. Heidelberg Berlin New York Tokyo: Springer; 2008. p. 110-9.

10. Gotoda T. Endoscopic resection of early gastric cancer. Gastric Cancer 2007;10:1-11.

11. Hurlstone DP, Atkinson R, Sanders DS, Thomson M, Cross SS, Brown S. Achieving R0 resection in the colorectum using endoscopic submucosal dissection. Br J Surg 2007;94:1536-42.

12. Gotoda T, Friedland S, Hamanaka H, Soetikno R. A learning curve for advanced endoscopic resection. Gastrointest Endosc 2005;62:866-7.

13. Coda S, Oda I, Gotoda T, Yokoi C, Kikuchi T, Ono H. Risk factors for cardiac stenosis and pyloric stenosis after endoscopic submucosal dissection. Endoscopy 2009;41:421-6.

14. Oka S, Tanaka S, Kaneko I, Mouri R, Hirata M, Kawamura T, et al. Advantage of endoscopic submucosal dissection compared with EMR for early gastric cancer. Gastrointest Endosc 2006; 64:877-83.

15. Probst A, Golger D, Arnholdt H, Messmann H. Endoscopic submucosal dissection of early cancers, flat adenomas, and submucosal tumors in the gastrointestinal tract. Clin Gastroenterol Hepatol 2009;7:149-55.

16. Dinis-Ribeiro M, Pimentel-Nunes P, Afonso M, Costa N, Lopes C, Moreira-Dias L. A European case-series of endoscopic submucosal dissection for gastric superficial lesions. Gastrointest Endosc 2009;69:350-5.

17. Catalano F, Trecca A, Rodella L, Lombardo F, Tomezzoli A, Battista S, et al. The modern treatment of early gastric cancer: our experience in an Italian cohort. Surg Endosc 2009; 23:1581-6.

18. Tanaka S, Oka S, Kaneko I, Hirata M, Mouri R, Kanao H, et al. Endoscopic submucosal dissection for colorectal neoplasia: possibility of standardization. Gastrointest Endosc 2007;66:100-7.

19. Saito Y, Uraoka T, Matsuda T, Emura F, Ikehara H, Massimo Y, et al. Endoscopic treatment of large superficial colorectal tumors: a case series of 200 endoscopic submucosal dissections (with video). Gastrointest Endosc 2007;66:966-73.

20. Tamegai Y, Saito Y, Masaki N, Hinohara C, Oshima T, Kogure E, et al. Endoscopic submucosal dissection: a safe technique for colorectal tumors. Endoscopy 2007;39:418-22

21. Zhou PH, Yao LQ, Qin XY. Endoscopic submucosal dissection for colorectal epithelial neoplasm. Surg Endosc 2009;23:154651. 